\title{
Computer modeling of the instability of a horizontal magnetic-fluid layer in a uniform magnetic field
}

\author{
V.G. Bashtovoi ${ }^{\mathrm{a}, *}$, O.A. Lavrova ${ }^{\mathrm{b}}$, V.K. Polevikov ${ }^{\mathrm{b}}$, L. Tobiska $^{\mathrm{c}}$ \\ ${ }^{a}$ Belarussian State Polytechnical Academy, 65 F.Skarina Ave., 220027 Minsk, Belarus \\ ${ }^{\mathrm{b}}$ Belarussian State University, 4 F.Skarina Ave., 220050 Minsk, Belarus \\ ${ }^{c}$ Otto-von-Guericke University of Magdeburg, Postfach 4120, D-39016 Magdeburg, Germany
}

\begin{abstract}
The stability of an initially flat layer of magnetic fluid that occupies the lower half-space under the influence of gravity and a uniform magnetic field normal to the plane surface is investigated numerically. Neutral curves of stability, critical parameters corresponding to the onset of instability, the equilibrium surface shape, and amplitudes of the perturbed surface are determined as functions of the magnetic-field intensity and magnetic-fluid properties.
\end{abstract}

(C) 2002 Published by Elsevier Science B.V.

Keywords: Magnetic fluid; Magnetic field; Surface instability; Computer modeling

\section{Introduction}

In the normal-field instability, a perpendicular, uniform magnetic field applied to an initially flat layer of magnetic fluid produces the spontaneous generation of an ordered pattern of surface protuberances when the field exceeds a critical value [1]. This effect has been studied both theoretically and experimentally [2], but its theoretical investigation is mainly restricted to the determination of the critical magnetic-field intensity in the case of small perturbations. A deeper theoretical insight is more difficult due to the necessity to solve a hydrostatic problem with unknown free-surface shape and the relevance of the magnetic field distortions on the surface that require an appropriate solving of the nonlinear Maxwell equations. The present paper is devoted to the numerical modeling of the phenomenon with regard to these factors.

\footnotetext{
*Corresponding author. Tel.: +375-017-32-85-90; fax: +375-017-31-00-52.

E-mail address: bashv@reoll.itmo.by (V.G. Bashtovoi).
}

\section{Statement of the problem}

The stability of a flat layer of magnetic fluid that occupies the lower half-space and that is bounded from above by a nonmagnetic gas is considered under the influence of gravity and a uniform magnetic field normal to the plane surface.

The present numerical study is based on the full system of Rosensweig's differential equations of ferrohydrostatics including Maxwell's [1]. The surface instability is examined in two cases: the case of twodimensional plane and axially symmetric perturbations. The development of such perturbations leads to the formation of periodic stationary structures on the free surface of the magnetic fluid, like linear rollers or peaks, when the intensity of the magnetic field exceeds a critical value $[1,2]$.

A dimensional analysis of the corresponding system of nonlinear partial differential equations shows that the problem is completely determined by the dimensionless parameters $\quad \chi=M_{\mathrm{s}} / H_{T}, \quad \lambda=a \sqrt{\rho g / \sigma}, \quad \mathrm{Si}=\mu_{0} M_{\mathrm{s}}^{2} /$ $(2 \sqrt{\rho g \sigma}), \mathrm{h}=H_{0} / H_{T}$, where $M_{\mathrm{S}}$ is the magnetization of saturation; $H_{T}$, the magnetic-field intensity at which the magnetization of the fluid is equal to $0.5 M_{\mathrm{s}}$ [3]; $a$, half of the wavelength of the perturbation; $\rho$ and $\sigma$, 
the density and the surface tension coefficient, respectively; $g$, the gravity acceleration; $\mu_{0}$, the magnetic permeability of vacuum; and $H_{0}$, the intensity of the external unperturbed magnetic field.

\section{Results}

The free-surface shape in equilibrium has been computed using the iteration-difference method described in Ref. [4], where the magnetic potential is approximated by finite elements.

The main results of the numerical study are presented in Figs. 1-4. A typical shape of the free surface of the magnetic fluid at a supercritical value of the magneticfield intensity in equilibrium is presented in Fig. 1. In addition, the corresponding structure of the magnetic field is shown by means of isolines of the magnetic potential $\Phi$ introduced by the relation $\mathbf{H}=\nabla \Phi$, where $\mathbf{H}$ is the magnetic-field intensity.

As shown in Fig. 2, the neutral curves of stability and the corresponding values of the critical magnetic-field intensity obtained numerically for axially symmetric and for plane perturbations do not differ very much and are in agreement with theoretical data.

Figs. 3 and 4 show some phenomena which cannot be obtained within the linear stability theory and determine the basic characteristics of the free-surface shape which

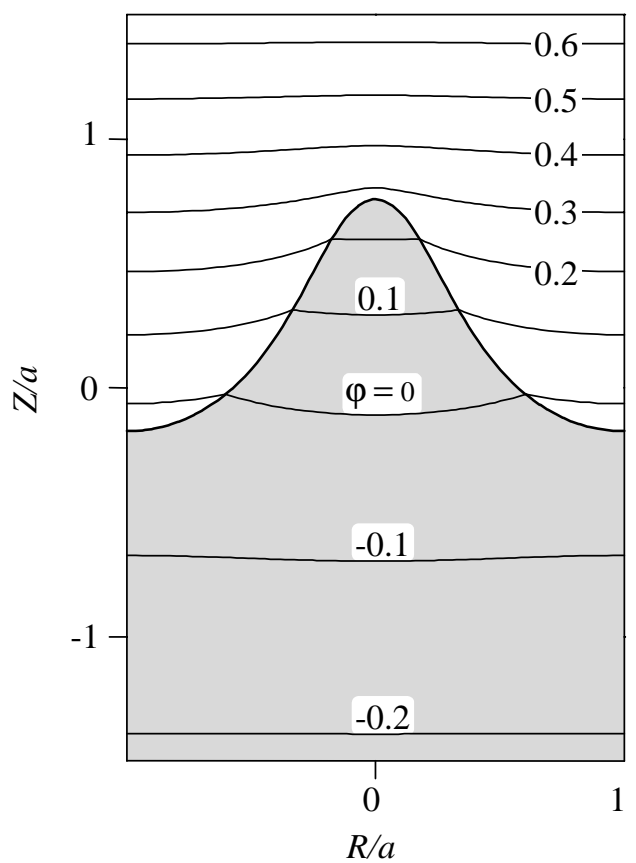

Fig. 1. Shape of the free surface and isolines of the dimensionless magnetic potential $\varphi=\Phi /\left(H_{T} a\right)$ at a supercritical value of the magnetic-field intensity. Axisymmetric problem with $\chi=$ $2.5, \lambda=\pi, \mathrm{Si}=100, \mathrm{~h}=0.45$.

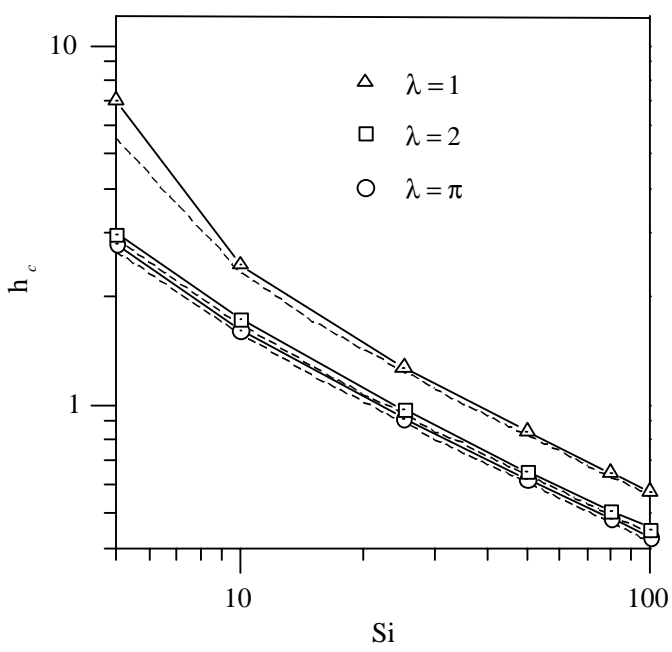

Fig. 2. Logarithmic dependence of the critical magnetic-field intensity on the parameter Si for the plane problem, $\chi=2.5$ and dimensionless wavelength $\lambda=1,2$ and $\pi$ (from top to bottom). Solid lines - numerical data; dashed lines - theoretical predictions.

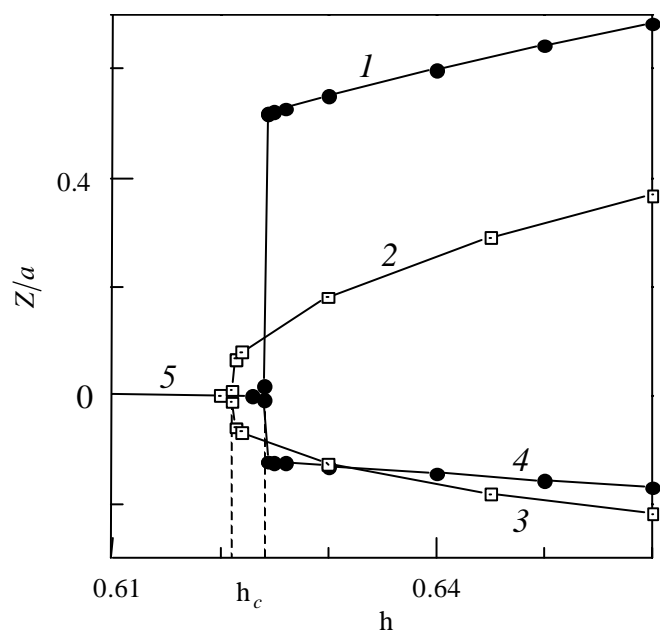

Fig. 3. Amplitude of the perturbed surface vs. the magnetic intensity parameter $\mathrm{h}$ for $\chi=2.5, \lambda=\pi$, and $\mathrm{Si}=50$. $(1,2)$ Peak top coordinate, $(3,4)$ foot coordinate, (5) horizontal surface position in the subcritical domain $\mathrm{h}<\mathrm{h}_{\mathrm{c}}$. $(1,4)$ Axisymmetric problem, $(2,3)$ plane problem.

appears beyond the instability. As can be seen from Fig. 3, perturbations of finite amplitude arise on the free surface when the magnetic-field intensity exceeds its critical value and then their amplitude grows monotonous with the increasing of field. Further, the peak top develops stronger compared to the foot. This difference is much more distinctive for the axially symmetric disturbances (peaks) than for the plane ones (rollers). 


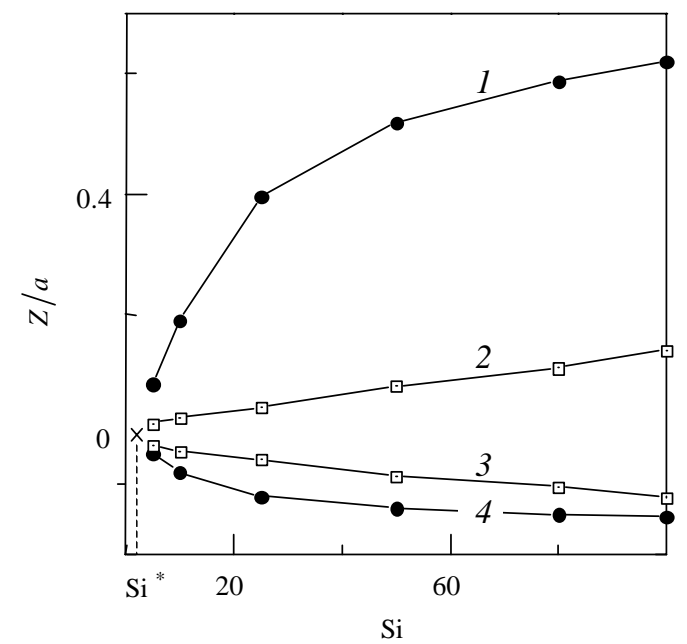

Fig. 4. Amplitude of the perturbed surface near the stability threshold $\left(\mathrm{h}_{\mathrm{c}}<\mathrm{h}<\mathrm{h}_{\mathrm{c}}+0.002\right)$ vs. the parameter Si for $\chi=2.5$, and $\lambda=\pi$. $(1,2)$ Top coordinate, $(3,4)$ foot coordinate. $(1,4)$ Axisymmetric problem, $(2,3)$ plane problem. $\mathrm{Si}^{*}=2$, critical value.

Moreover, under the same conditions the amplitude of the axially symmetric perturbations of the surface (peaks) is significantly greater than for the plane ones (rollers).
In this context the initial amplitude of the surface perturbations near the stability threshold is of particular interest. The dependence of the amplitude on the fluid properties is illustrated in Fig. 4. As expected, with the strengthening of magnetic properties of the fluid (with increasing magnetization of saturation) the initial amplitude of surface perturbations increases considerably. Finally, Fig. 4 also illustrates the well-known experimental fact that if the fluid magnetization is less than a limiting value $(\mathrm{Si}<2$ for considered problems), the instability of the fluid surface does not appear at any value of the magnetic-field intensity.

\section{References}

[1] R.E. Rosensweig, Ferrohydrodynamics, Cambridge University Press, Cambridge, 1985.

[2] B. Berkovski, V. Bashtovoi (Eds.), Magnetic Fluids and Applications Handbook, Begel House Inc. Publ., New York, 1996.

[3] A.N. Vislovich, Magnetohydrodynamics, No. 2, 1990.

[4] V.K. Polevikov, Preprint No. 10, Otto-von-Guericke University of Magdeburg, 1998. 